\title{
The triplet state of the ladder-type methyl-poly(p-phenylene) as seen by pulse radiolysis-energy transfer
}

\author{
A.P. Monkman ${ }^{\text {a, }}$, H.D. Burrows ${ }^{b}$, I. Hamblett ${ }^{c}$, S. Navaratnam ${ }^{\text {d, }}$, \\ U. Scherf ${ }^{\mathrm{e}}$, C. Schmitt ${ }^{\mathrm{e}}$ \\ a Department of Physics, University of Durham, Durham, DH1 3LE, UK \\ b Departamento de Química, Universidade de Coimbra, 3049 Coimbra, Portugal \\ ${ }^{\mathrm{c}}$ Department of Chemistry, University of Manchester, Manchester M13 9PL, UK \\ ${ }^{\mathrm{d}}$ Multidisciplinary Research and Innovation Centre, North East Wales Institute, Wrexham LL11 2AW, UK \\ ${ }^{\mathrm{e}}$ Max-Planck-Institut für Polymerforschung, Ackermannweg 10, D-55128 Mainz, Germany
}

Received 4 July 2000; in final form 10 July 2000

\begin{abstract}
The lowest triplet state of a ladder poly( $p$-phenylene) (MeLPPP) has been produced by sensitised energy transfer following pulse radiolysis of benzene solution, and absorbs at $1.34 \mathrm{eV}$ with a lifetime $\geqslant 170 \mu \mathrm{s}$. An $\mathrm{S}_{0}-\mathrm{T}_{1}$ energy separation of $2.15 \pm 0.07 \mathrm{eV}$ was determined, in excellent agreement with recent phosphorescence measurements, confirming the validity of the technique for the determination of the triplet energies of conjugated polymers. At high triplet populations intrachain triplet-triplet annihilation (TTA), leading to delayed fluorescence (DF) occurs. The DF spectrum is in good agreement with the prompt fluorescence and the DF kinetics support a TTA mechanism. (C) 2000 Elsevier Science B.V. All rights reserved.
\end{abstract}

\section{Introduction}

Although estimates differ on the relative yields of production of singlet and triplet excited states by charge recombination in the electroluminescence of conjugated organic polymers [1-4], it is generally accepted that triplet state formation is one of the major causes of efficiency loss in these systems when they are used in light emitting devices [5]. Triplet states are also important for potential applica-

\footnotetext{
* Corresponding author. Fax: +44-191-374-3848; e-mail: a.p.monkman@durham.ac.uk
}

tions of conjugated polymers as lasers [6], and knowledge of the energy of the lowest triplet state is a prerequisite for the design of high efficiency light emitting devices based on triplet energy capture and transfer [7]. However, although information on triplet states in these systems has been obtained by a variety of techniques, including optically detected magnetic resonance [8,9], photoinduced absorption $[10,11]$ and photomodulation spectroscopy [12], only in the case of the ladder-type methyl-substituted poly ( $p$-phenylene), MeLPPP (Fig. 1) has unambiguous evidence been presented for phosphorescence in a conjugated organic polymer [13]. A previous report of phosphorescence from poly(3-hexylthiophene) 


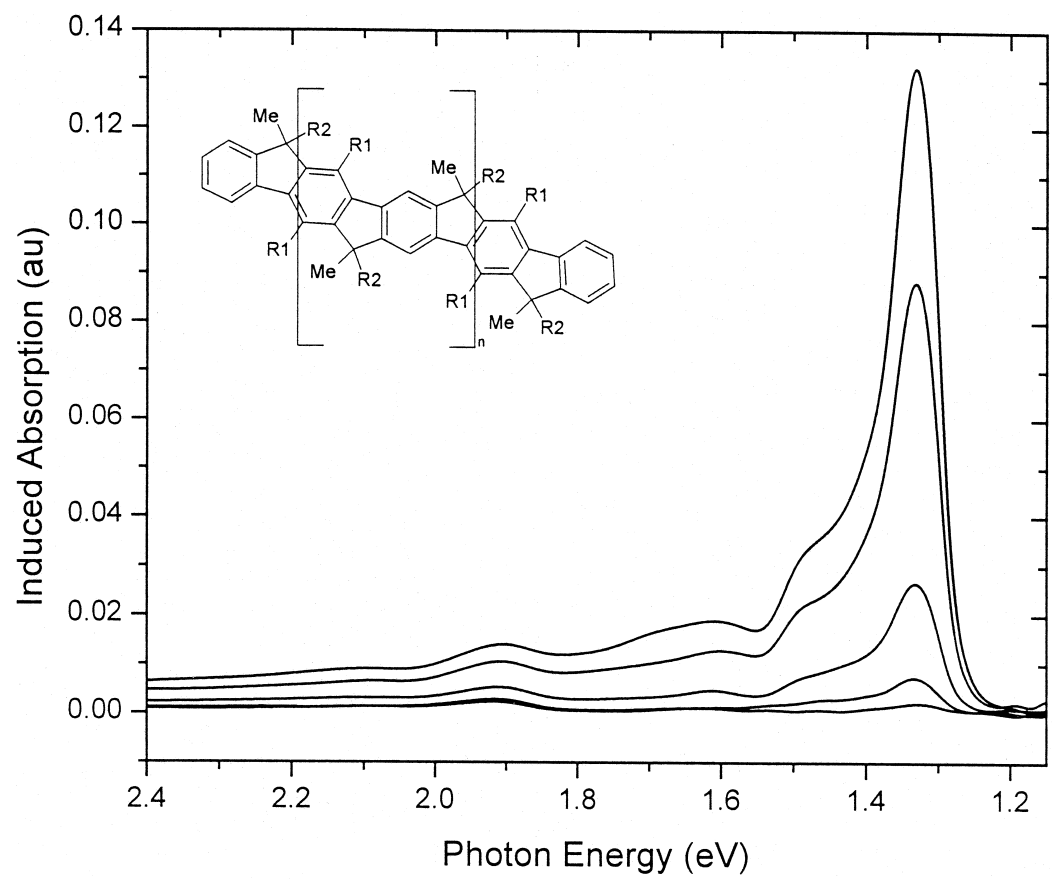

Fig. 1. Transient absorption spectra observed at various times $(25,50,100,150$ and $250 \mu \mathrm{s})$ following pulse radiolysis of an argon saturated solution of MeLPPP $(40 \mathrm{mg} / 1)$ in benzene in the presence of biphenyl $(0.01 \mathrm{M})$. The structure of MeLPPP is shown in the inset, R1 = $n-\mathrm{C}_{6} \mathrm{H}_{13}$ and $\mathrm{R} 2=1,4-\left(\mathrm{C}_{6} \mathrm{H}_{4}\right)-n-\mathrm{C}_{10} \mathrm{H}_{21}$.

[14], has been questioned [13], and the existence of phosphorescence from poly(thiophenes), or other polymers, awaits confirmation.

Triplet-triplet energy transfer following pulse radiolysis of solutes $(\mathrm{S})$ in aromatic solvents, such as benzene $(\mathrm{Bz})$, in the presence of appropriate energy acceptors (A) is an excellent technique for selective creation and study of excited triplet states $[15,16]$. The general reactions scheme is

$\mathrm{Bz}+\mathrm{e}^{*} \leadsto{ }^{1} \mathrm{Bz}^{*}+{ }^{3} \mathrm{Bz}^{*}+\mathrm{e}^{*}$

${ }^{1} \mathrm{Bz}^{*} \rightarrow{ }^{3} \mathrm{Bz}^{*}$

${ }^{3} \mathrm{Bz}^{*}+\mathrm{A} \rightarrow \mathrm{Bz}+{ }^{3} \mathrm{~A}^{*}$

${ }^{3} \mathrm{~A}^{*}+\mathrm{S} \rightarrow \mathrm{A}+{ }^{3} \mathrm{~S}^{*}$

where $[\mathrm{Bz}] \gg[\mathrm{A}] \gg[\mathrm{S}]$

We have previously shown that this technique can be applied to study the triplet states of conjugated organic polymers in solution [6,17]. In addition to its use in obtaining spectral and kinetic information, it is possible, by varying the (triplet) energy of $\mathrm{A}$, to determine the $\mathrm{S}_{0}-\mathrm{T}_{1}$ separation in the conjugated polymer. We have used this method to estimate the triplet energies of a wide range of some of the most commonly used conjugated organic polymer $[6,17,18]$. Triplet-triplet energy transfer following photoexcitation has also been used to determine the triplet energy of a poly(aryleneethynylene) [19].

Triplet-triplet energy transfer is subject to both energetic [20,21] and kinetic [22] restraints, and it is important to compare results on triplet energies obtained using this technique with absolute data from phosphorescence measurements to confirm the validity of the method. We have, therefore, applied the method to MeLPPP, whose $\mathrm{S}_{0}-\mathrm{T}_{1}$ energy separation has previously been determined to be $2.05 \mathrm{eV}$ by phosphorescence from thin films of the polymer at $77 \mathrm{~K}[13]$.

\section{Experimental details}

The synthesis and purification of MeLPPP have previously been reported $[23,24]$. The polymer had a 
molecular weight $M_{n} \approx 6.9 \times 10^{4}$ and a polydispersity ca. 2.8. Pulse radiolysis experiments were carried out at the Free Radical Research Facility of the Paterson Institute for Cancer Research (Manchester, UK) using, typically $50 \mathrm{~ns}$, ca. 7.5 Gray pulses from a $10 \mathrm{MeV}$ electron linear accelerator. Transient absorption difference spectra were obtained using a Xenon source and gated diode array or photomultiplier for detection. General details have been reported elsewhere $[17,25]$.

\section{Results and discussion}

Following pulse radiolysis of argon saturated solutions of biphenyl (0.01 M) and MeLPPP (40 mg/l) in benzene, an initial transient absorption, due to the biphenyl triplet state $[15,16,26]$, was observed at $360 \mathrm{~nm}$. This was seen to decay, and to be replaced with the grow-in of a new absorption at $925 \mathrm{~nm}(1.34 \mathrm{eV})$, assigned to the MeLPPP triplet state (Fig. 1). Vardeny and co-workers $[12,27]$ have reported a triplet-triplet absorption in thin films for a similar poly ( $p$-phenylene) at $1.3 \mathrm{eV}$ using photomodulation spectroscopy. The triplet state was seen to decay by good first-order kinetics with a lifetime $\tau \geqslant 170 \mu \mathrm{s}$

In the presence of $0.4 \% \quad \mathrm{O}_{2} \quad(35 \mu \mathrm{M})$, the decay of the transient absorption at $1.35 \mathrm{eV}$ in biphenyl $(0.01 \mathrm{M})$, benzene solutions, was faster, in agreement with assignment to the ${ }^{3} \mathrm{MeLPPP}^{*}$ state. Assuming that the quenching by oxygen follows pseudo first-order kinetics, a rate constant for bimolecular recombination of $k=7 \times 10^{8} \mathrm{M}^{-1} \mathrm{~s}^{-1}$ was determined for this reaction. This is slightly slower than the rate of $\mathrm{O}_{2}$ quenching of poly $(p$ phenylenevinylene) triplet states [17,28], but is within the range typically observed for quenching aromatic triplet states by molecular oxygen. Quenching in these systems can lead to the formation of singlet oxygen via the energy transfer reaction

${ }^{3} \mathrm{~S}^{*}+\mathrm{O}_{2}\left({ }^{3} \Sigma_{\mathrm{g}}^{-}\right) \rightarrow \mathrm{S}+{ }^{1} \mathrm{O}_{2}^{*}\left({ }^{1} \Delta_{\mathrm{g}}\right)$

and the singlet oxygen detected by its phosphorescence at $1270 \mathrm{~nm}$. Following irradiation of an aerated solution of MeLPPP in benzene with a fre- quency tripled pulse $(355 \mathrm{~nm})$ from a Nd:YAG laser, emission was observed at $1270 \mathrm{~nm}$, confirming that ${ }^{3}$ MeLPPP ${ }^{*}$ does indeed sensitise the generation of singlet oxygen. This decayed with a lifetime $\tau=29$ $\pm 2 \mu \mathrm{s}$, in reasonable agreement with the reported lifetime for singlet oxygen in benzene (32 $\mu$ s [29]). The quantum yield of singlet oxygen formation was determined as described elsewhere [30] by comparison of the initial emission intensity for optically matched solutions at the excitation wavelength $\left(\mathrm{A}_{355}\right.$ $=0.5)$ with a standard. $1 H$ Phenalen-1-one was used as standard, taking $\Phi_{\Delta}=0.93$ in benzene [31]. Although the plot of singlet oxygen phosphorescence intensity as a function of laser power was not linear, and tends to plateau at high laser intensities, it was possible to estimate from the initial slope a singlet oxygen yield $0.085 \pm 0.018$ for singlet oxygen production sensitised by MeLPPP in benzene solution. The reason for the non-linearity of the phosphorescence as a function of excitation dose is not completely clear, but is probably associated with other triplet deactivation pathways, such as triplettriplet annihilation, occurring at high intra-chain triplet densities. This will be discussed later. As with PPV based polymers [17,28], singlet oxygen reactions are likely to be important in the degradation of ladder type $p$-phenylene polymers in non-encapsulated devices. This value of the singlet oxygen yield also gives a lower limit for the $\mathrm{S}_{1}-\mathrm{T}_{1}$ intersystem crossing in photoexcited MeLPPP in benzene solutions.

The fact that molecular oxygen quenches the MeLPPP triplet state, produced by energy transfer from biphenyl, indicates that it has an energy between singlet oxygen $\left({ }^{1} \Delta_{\mathrm{g}}, 0.94 \mathrm{eV}\right.$ [26]) and triplet biphenyl (2.84 eV [26]). To determine the energy more accurately, a variety of sensitisers were used to transfer the energy to MeLPPP on pulse radiolysis of argon saturated benzene solutions. It was found that while fluorenone $(2.19 \mathrm{eV})$ and sensitisers with higher (triplet) energies transfer triplet energy efficiently, only very low yields of the MeLPPP triplet state could be produced by pyrene sensitisation, while compounds with lower energy triplet states did not transfer energy to the polymer. We can, thus, estimate the triplet energy of MeLPPP in benzene solution as $2.15 \pm 0.07 \mathrm{eV}$. The value reported from phosphorescence measurements for MeLPPP in thin 
films at $77 \mathrm{~K}$ is $2.05 \mathrm{eV}$ [13]. Considering that differences of around $100 \mathrm{meV}$ exist between singlet energies of these polymers in solution and films [6], the agreement between the two techniques is excellent, and confirms the validity of the pulse radiolysis-energy transfer technique for determining triplet energies of conjugated organic polymers.

Under the conditions used in our pulse radiolysis experiments, it is likely that there is more than one triplet state per polymer chain. We have previously shown [32] that delayed fluorescence (DF), resulting from triplet-triplet ( $\mathrm{T}-\mathrm{T})$ annihilation, can be observed in such systems. DF via $\mathrm{T}-\mathrm{T}$ annihilation has previously been inferred from observations following photoexcitation of films or frozen solutions of poly ( $p$-phenylenevinylene) or poly( $p$-phenylenethynylene) [33]. However, time resolved measurements clearly show that for direct optical excitation, DF arises from charge state recombination [13].
Following pulse radiolysis of argon saturated solutions of MeLPPP (10 mg/l) and biphenyl (0.01 M) in benzene, an emission in the $2.8-2.2 \mathrm{eV}$ region was seen to grow-in within $10 \mu$ s and to decay over ca. $150 \mu \mathrm{s}$. The time dependent DF spectrum, corrected for the response of the detection system, is shown in Fig. 2, and is in excellent agreement with the reported prompt fluorescence spectra of MeLPPP $[1,13,34]$. Delayed fluorescence from charge carrier recombination has previously been observed with films of these ladder polymers [1,34], and also show a second broad feature in the $2.25-2.1 \mathrm{eV}$ region assigned to an excimer-like emission. This broad emission was absent in our measurements. This is reasonable, since at the concentrations used in the pulse radiolysis experiments $\left(\approx 2 \times 10^{-7} \mathrm{M}\right.$ polymer) we are dealing, effectively, with isolated polymer chains [32], whereas in the films there are greater possibilities for inter-chain interactions. We

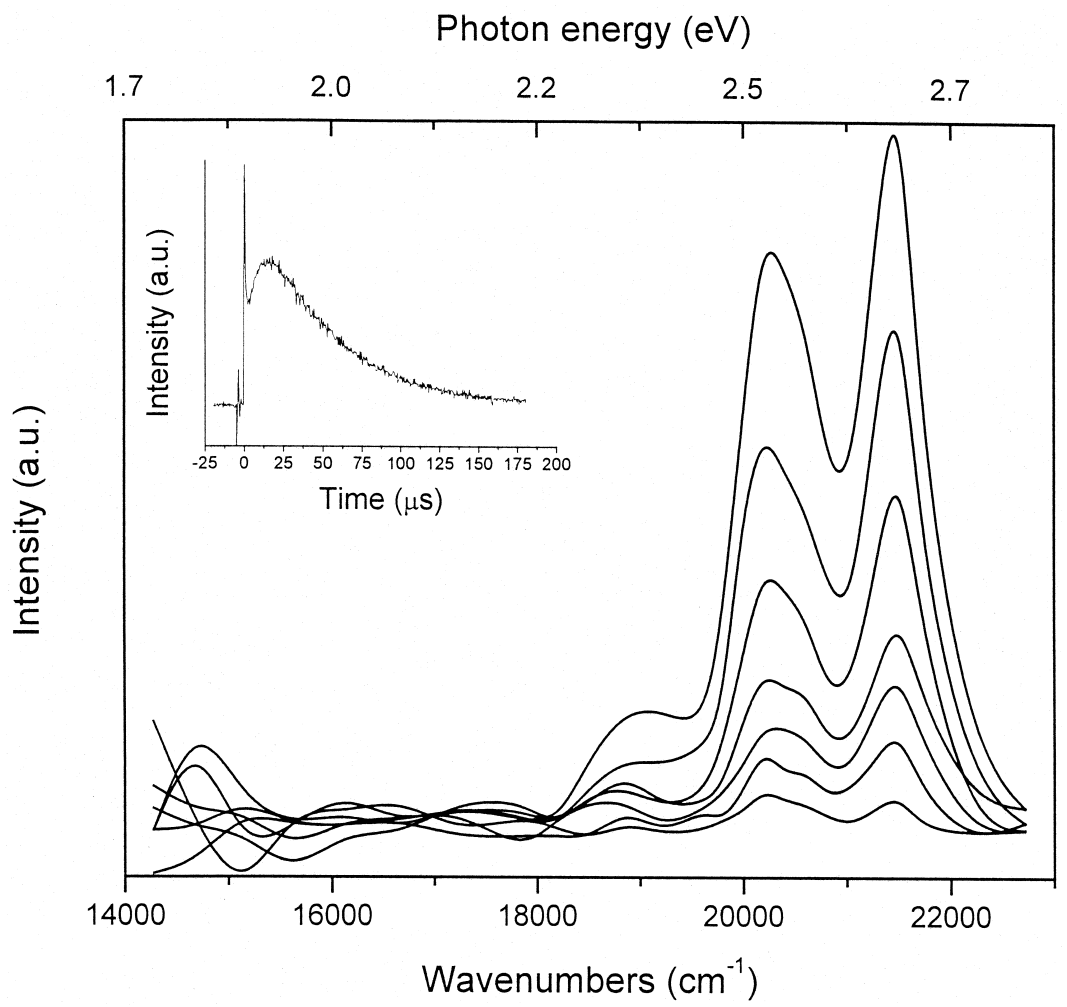

Fig. 2. Delayed fluorescence (DF) spectra, corrected for the sensitivity of the detection system, observed at various times (18, 40, 60, $80,100,120$ and $160 \mu \mathrm{s})$ after excitation of argon saturated solutions of MeLPPP (10 mg/1), biphenyl (0.01 M) in benzene with ca. 7.5 Gray electron-beam pulses from a linear accelerator. The inset shows the decay kinetics of the DF signal measured at $2.56 \mathrm{eV}$. 
looked unsuccessfully for phosphorescence following pulse radiolysis of solutions at room temperature, see the spectral region below $2.2 \mathrm{eV}$ in Fig. 1 . Again, this negative result is not surprising, as even at low temperature the phosphorescence yield is low, and decreases even more upon increasing temperature [13]. If there is any phosphorescence in these systems, it is below the limits of our detection system.

The time dependence of the DF was monitored as a function of the radiation dose, and follow similar behaviour to the other conjugated polymers we have studied [32]. In particular, the rate of DF decay increases with dose, as expected for bimolecular $\mathrm{T}-\mathrm{T}$. For example, the lifetime decreases from ca 42 $\mu \mathrm{s}$ to $13 \mu \mathrm{s}$ on increasing the dose from ca 15 to 27 Grays

As already stated, in addition to $\mathrm{T}-\mathrm{T}$ annihilation, an alternative mechanism for $\mathrm{DF}$, involving charge recombination of geminate charge pairs, has been suggested for the emission observed on photoexcitation of films of MeLPPP $[1,34]$. While it is generally difficult to differentiate between the triplet-triplet annihilation and charge recombination mechanisms for DF [1], we believe our kinetic data is more consistent with the $\mathrm{T}-\mathrm{T}$ annihilation mechanism. Pulse radiolysis experiments in chloroform and tetrahydrofuran show that the positive and negative charge carriers derived from MeLPPP have an absorption band at $650 \mathrm{~nm}(1.9 \mathrm{eV})$ with a weaker feature at $590 \mathrm{~nm}(2.1 \mathrm{eV})$ [35]. The spectrum observed on pulse radiolysis of solutions of MeLPPP in benzene in the presence of biphenyl (Fig. 1) does show weak absorption at $1.9 \mathrm{eV}$, however the decay of this feature parallels that of the main triplet absorption at $1.34 \mathrm{eV}$ suggesting that it too is associated with the $\mathrm{T}-\mathrm{T}$ absorption. The $\mathrm{T}-\mathrm{T}$ mechanism is likely to be favoured in our system by the high density of triplet states on the isolated polymer chains, and the fact that there are effectively no inter-chain interactions.

If we assume that triplets move by hopping [32], and that the MeLPPP is a rigid rod, from the observed delayed fluorescence decay we can estimate an order of magnitude for the triplet hopping rate of $10^{5}-10^{6} \mathrm{~nm} \mathrm{~s}^{-1}$. More detailed studies are in progress to quantify the kinetics of intra-chain triplet hopping and $\mathrm{T}-\mathrm{T}$ annihilation on isolated chains of conjugated polymers

\section{Acknowledgements}

We thank the Paterson Institute for Cancer Research Free Radical Research Facility (Manchester, UK) for access to the pulse radiolysis equipment, and Dr. Donald Allan and Brian Hodgson for their ability to keep this facility working for as long as they did against increasingly insurmountable odds. Financial support from EPSRC (GR/M86040), PRAXIS XXI (project 2/2.1/QUI/411/95) and British Council/CRUP (project B-52/00) is gratefully acknowledged.

\section{References}

[1] Yu.V. Romanovskii, A. Gerhard, B. Schweitzer, R.I. Personov, H. Bässler, Chem. Phys. 249 (1999) 29.

[2] M.A. Baldo, D.F. O'Brien, M.E. Thompson, S.R. Forrest, Phys. Rev. B 60 (1999) 14422.

[3] Y. Cao, I.D. Parker, C. Yu, C. Zhang, A.J. Heeger, Nature 397 (1999) 414.

[4] Z. Shuai, D. Beljonne, R.J. Silbey, J.L. Brédas, Phys. Rev. Lett. 84 (2000) 131

[5] R.H. Friend, R.W. Gymer, A.B. Holmes, J.H. Burroughes, R.N. Marks, C. Taliani, D.D.C. Bradley, D.A. Dos Santos, J.L. Brédas, M. Lögdlund, W.R. Salaneck, Nature 397 (1999) 121.

[6] A.P. Monkman, H.D. Burrows, L.E. Horsburgh, L.J. Hartwell, M. da G. Miguel, I. Hamblett, S. Navaratnam, Proc. SPIE 3797 (1999) 109.

[7] M.A. Baldo, M.E. Thompson, S.R. Forrest, Nature 403 (2000) 750.

[8] L.S. Swanson, J. Shinar, K. Yoshino, Phys. Rev. Lett. 65 (1990) 1140.

[9] V. Dykonov, G. Rösler, M. Schwoerer, E.L. Frankevich, Phys. Rev. B 56 (1997) 3852.

[10] R.H. Friend, D.D.C. Bradley, P.D. Townshend, J. Phys. D: Appl. Phys. 20 (1987) 1367

[11] G. Cerrullo, S. Stagira, M. Nisoli, S. De Silvestri, G. Lanzani, G. Kranzelbinder, W. Graupner, G. Leising, Phys. Rev. B 57 (1998) 12806

[12] M. Wohlgenannt, C.P. An, Z.V. Vardeny, J. Phys. Chem. B 104 (2000) 3846.

[13] Yu.V. Romanovskii, A. Gerhard, B. Schweitzer, U. Scherf, R.I. Personov, H. Bässler, Phys. Rev. Lett. 84 (2000) 1027.

[14] B. Xu, S. Holdcroft, J. Am. Chem. Soc. 115 (1993) 8447.

[15] E.J. Land, Proc. Roy. Soc. A 305 (1968) 457.

[16] R.V. Bensasson, E.J. Land, T.G. Truscott, Excited States and Free Radicals in Biology and Medicine, Oxford University Press, Oxford, 1993.

[17] A.P. Monkman, H.D. Burrows, M. da G. Miguel, I. Hamblett, S. Navaratnam, Chem. Phys. Lett. 307 (1999) 303.

[18] A.P. Monkman, H.D. Burrows, L.J. Hartwell, L.E. Horsburgh, I. Hamblett, S. Navaratnam, Phys. Rev. Lett. submitted for publication. 
[19] K.A. Walters, K.D. Ley, K. Schanze, Chem. Commun., 1998, p. 1115.

[20] T. Förster, Discuss. Faraday Soc. 27 (1959) 7.

[21] D.L. Dexter, J. Chem. Phys. 21 (1953) 836.

[22] K. Sandros, Acta Chem. Scand. 18 (1964) 2355.

[23] U. Scherf, K. Mullen, Makromol. Chem. Rapid Commun. 12 (1991) 489.

[24] U. Scherf, A. Bohnen, K. Mullen, Makromol. Chem. 193 (1992) 1127.

[25] J. Butler, B.W. Hodgson, B.M. Hoey, E.J. Land, J.S. Lea, E.J. Lindley, F.A.P. Rushton, A.J. Swallow, Radiat. Phys. Chem. 34 (1989) 633.

[26] S.L. Murov, I. Carmichael, G.L. Hug, Handbook of Photochemistry, 2nd ed., Marcel Dekker, New York, 1993.

[27] M. Wohlgenannt, W. Graupner, G. Leising, Z.V. Vardeny, Phys. Rev. Lett. 82 (1999) 3344.
[28] R.D. Scurlock, B. Wang, P.R. Ogilby, J.R. Sheats, R.L. Clough, J. Am. Chem. Soc. 117 (1995) 10194.

[29] A.A. Gorman, A.A. Krasnovsky, M.A.J. Rodgers, J. Phys. Chem. 95 (1991) 598.

[30] J.P. Keene, D. Kessel, E.J. Land, R.W. Redmond, T.G. Truscott, Photochem. Photobiol. 43 (1986) 117.

[31] E. Oliveros, P. Suardi-Murasecco, T. Aminian-Saghafi, A.M. Braun, H.-J. Hansen, Helv. Chim. Acta 74 (1991) 79.

[32] A.P. Monkman, H.D. Burrows, I. Hamblett, S. Navaratnam, Phys. Rev. Lett., submitted for publication.

[33] J. Partee, E.L. Frankevich, B. Uhlhorn, J. Shinar, Y. Ding, T.J. Barton, Phys. Rev. Lett. 82 (1999) 3673.

[34] B. Schweitzer, V.I. Arkhipov, H. Bässler, Chem. Phys. Lett. 304 (1999) 365.

[35] H.D. Burrows, A.P. Monkman, I. Hamblett, S. Navaratnam, to be published. 